barriers of adherence to home exercise programs in patients with OA by qualitative research methods.

Objectives: Aim of our study was to investigate the barriers and facilitators for adherence of home-based exercises for knee osteoarthritis management from the perspective of physiotherapists and patients.

Methods: A Qualitative study by using focus groups discussions and semi-structured interviews were designed to investigate the barriers and facilitators to home-based exercise program adherence for OA. Two researchers facilitated focus group interview. Participants of focus group members were eight physiotherapists (PT) working with OA with different experience levels. Third researcher conduct the interviews which lasted 30-60 minutes with patients (patients with knee $O A, n=5$ ages $>50$ ). Data were audio recorded, transcribed verbatim and thematically analyzed with NVIVO 12 software. Three researchers conducted the thematic analysis to ensure the validity.

Results: In total, 25 main themes from the focus group discussions and interviews were determined. Major barrier themes from focus group were (a) beliefs to exercise benefits (b) patient education and (c)fear avoidance beliefs on exercise; from the interviews were (a)negligence of self-management (b) fatigue and (c)patient education. Patients and therapists all agreed for patient education is one of the most important factors for home EA. Patients wanted to get education on arthritis management. A patient said: "Actually, the clinicians should give information more deeply. I don't know which is correct for me after therapy, resting or moving?" Major facilitator themes from the focus group were (a)motivation from PT (b)client-centred exercise (c)digital technology; from interviews were (a) motivational approaches of therapists (wats-up messages) (b) having pain and (c)patient education for disease management. Therapists agreed on that personalized exercise is the most important facilitator. A therapist commented, "If the personalized exercise given the patient with correct intensity and repetitions, I don't think that patients would not do their home exercises."

Conclusion: This is the first qualitative study about exercise adherence in knee osteoarthritis in Turkey. It has been determined that the lack of education and motivation are the most important barriers. More studies are needed to examine the factors affecting EA for patients with OA. In future studies, implementations to increase home EA on Turkish patients with OA should be investigated by qualitative research methods.

References:

[1] MacKay C. Qualitative study exploring the factors influencing physical therapy management of early knee osteoarthritis in Canada. BMJ 2018 open.

[2] Carmona-Terés, Understanding knee osteoarthritis from the patients' perspective: a qualitative study." BMC musculoskeletal disorders 18.1 (2017): 225.

[3] Marlene F.Exercise for osteoarthritis of the knee: a Cochrane systematic review. British journal of sports medicine 2015 49(24).

Disclosure of Interests: None declared

DOI: 10.1136/annrheumdis-2020-eular.3924

\section{SAT0618-HPR PATIENTS WITH INFLAMMATORY MYOPATHIES WHO DO NOT REACH HEALTH ENHANCING LEVELS OF PHYSICAL ACTIVITY REPORT HIGHER LEVELS OF ANXIETY AND DEPRESSION - A CROSS-SECTIONAL STUDY OF SELF-REPORTED DATA}

K. Andreasson ${ }^{1,2}, \mathrm{H}$. Sandlund ${ }^{3}, \mathrm{H}$. Alexanderson ${ }^{1,2}$. ${ }^{1}$ Karolinska Institute, Department of Medicine, Stockholm, Sweden; ${ }^{2}$ Karolinska University Hospital, Unit of Physical Therapy, Stockholm, Sweden; ${ }^{3}$ Karolinska University Hospital, Unit of Rheumatology, Stockholm, Sweden

Background: The adult idiopathic inflammatory myopathies (IIM) comprise dermatomyositis (DM), polymyositis (PM), immune-mediated necrotizing myopathy (IMNM), antisynthetase syndrome (ASS), overlap myositis and inclusion body myositis (IBM). Impaired physical capacity, self-reported fatigue and pain are common features in IIM. Quality of life is reduced compared to population-based reference values. To our knowledge self-reported levels of physical activity has not been studied in patients with IIM. Further, anxiety and depression are common in other rheumatic diseases, such as SLE, but is less studied in IIM, and not previously in relation to levels of physical activity. There is evidence for symptom reducing effects of exercise for patients suffering from depression (1).

Objectives: The objective of this study is to assess the levels of self-reported physical activity, depression and anxiety amongst adult patients with IIM. A further aim is to evaluate differences in anxiety/depression based on levels of physical activity as well as to analyze relationships between physical activity and anxiety/depression.

Methods: All patients with IIM visiting the Rheumatology clinic at Karolinska University Hospital in Solna between February 2019 and January 2020 where asked to fill in questionnaires about their levels of physical activity for the last seven days using the International Physical Activities Questionnaire - short form (IPAQ), and anxiety and depression using Hospital Anxiety and Depression Scale (HADS). The myositis team nurse distributed the questionnaires. Spearman's rho was used for correlation analysis. Kruskal-Wallis test and post-hoc adjustment with Bonferroni correction was used to analyze group differences. HADS is scored in two separate scales, one for depression (HADS-D) and one for anxiety (HADS-A). The cut-off value for probable depression or anxiety is $\geq 8$ of a maximum of 21 per scale (2). IPAQ-results was scored as 1 (low, $150 \mathrm{~min} / \mathrm{w}$ ), 2 (moderate, $\geq 150 \mathrm{~min} / \mathrm{w}$ - health-enhancing levels of physical activ ity, HEPA, according to WHO) and 3 (high, $\geq 300 \mathrm{~min} / \mathrm{w}$ ).

Results: A total of 61 patients answered the questionnaires. 52 (85\%) of the patients reported to reach HEPA and 24 of these patients reported to be active on a high level. 22 patients (36\%) scored probable anxiety or depression, with six scoring $\geq 8$ for both depression and anxiety. Patients with low levels of physical activity (IPAQ-1) scored significantly higher anxiety and depression compared to those reaching HEPA (IPAQ-2 and IPAQ-3) $p<0.0001-0.020$. The correlation between physical activity and depression (Fig. 1) was $r s=-0.48(-0.66 ;-0.26)$ and between physical activity and anxiety (Fig. 2), rs=-0.27 (-0.49; -0.02).

Conclusion: Self-reported data indicates that most patients with IIM in this sample reached HEPA level or higher. Patients who do not reach HEPA score significantly higher anxiety and depression compared to those reaching HEPA. However, levels of physical activity correlates moderately to depression and weakly to anxiety. The number of patients who reached HEPA is high compared to studies in rheumatoid arthritis or the general population. This could be explained by frequent visits to physical therapists early in the disease and yearly check-ups with a focus on exercise and physical activity. Further the inter-professional myositis team also has a focus on exercise and the importance of everyday physical activity. This is cross-sectional, self-reported data and longitudina studies are needed also including objective measures. This is preliminary data with data collection ongoing throughout 2020

References:

[1] Craft, LL et al. The benefits of exercise for the clinically depressed. Prim Care Companion J Clin Psychiatry. 2004;6(3):104-111

[2] Zigmond, AS et al. The hospital anxiety and depression scale. Acta Psychiat. Scand. 1983;67(6):361-70

Disclosure of Interests: None declared

DOI: 10.1136/annrheumdis-2020-eular.938

\section{SAT0619-HPR AN AUDIT OF GLUCOCORTICOID PRESCRIPTION IN PATIENTS WITH GIANT CELL ARTERITIS}

H. H. Ariff ${ }^{1}$, A. Awisat ${ }^{1}$, J. Arnold ${ }^{1}$, H. Al Ani ${ }^{1}$, L. O'neill ${ }^{1}$, M. P. Rodriguez ${ }^{2}$, R. Luqmani ${ }^{1} .{ }^{1}$ Nuffield Orthopaedic Centre, Rheumatology, Oxford, United Kingdom; ${ }^{2}$ Leeds Institute of Health Sciences, Leeds, United Kingdom

Background: Giant cell arteritis (GCA) is treated with high dose glucocorticoids

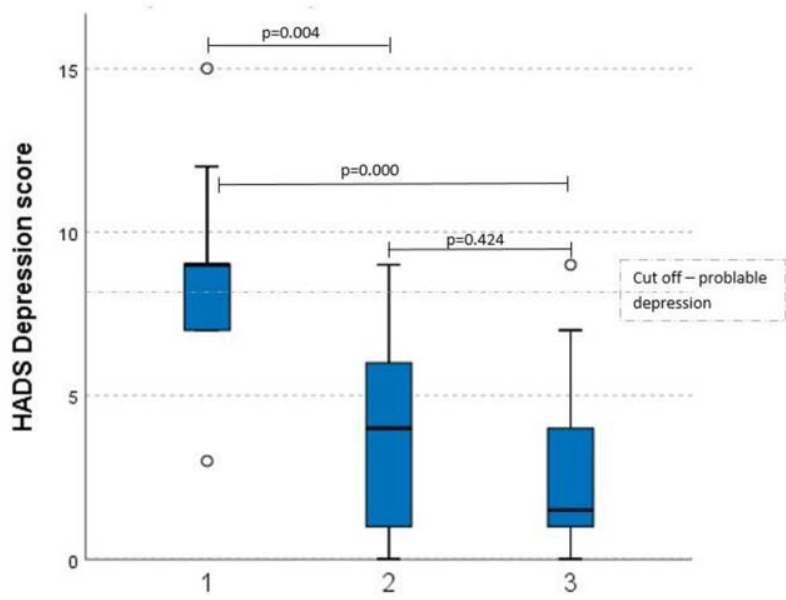

Figure 1. IPAQ level

1-low physical activity, not reaching health-enhancing levels of physical activity (HEPA), 2moderate physical activity, reaching HEPA, 3-high physical activity, surpasses HEPA

and progressively reduced over months to years.

Objectives: We undertook an audit to evaluate self-reported adherence to the original recommended glucocorticoid course and explored reasons for any variation. Methods: We recruited patients attending a single rheumatology department over 18 months. Respondents were given two self- administered questionnaires 